\title{
Rapid blood flow computation on Digital Subtraction Angiography: Preliminary results
}

George Bourantas ${ }^{1}$, Grand Roman Joldes ${ }^{1}$, Konstantinos Katsanos ${ }^{2,3}$, George C. Kagadis $^{4,5}$, Adam Wittek ${ }^{1}$, Karol Miller ${ }^{1,6}$

${ }^{1}$ Intelligent Systems for Medicine Laboratory,

The University of Western Australia,

Crawley-Perth 6009, Western Australia, Australia

${ }^{2}$ The Department of Interventional Radiology, Patras University Hospital, School of Medicine, 26504 Rion, Greece

${ }^{3}$ The Department of Interventional Radiology, Guy's and St. Thomas' Hospitals, NHS Foundation Trust, King's Health Partners, London SE1 7EH, UK

${ }^{4}$ Department of Medical Physics, School of Medicine, University of Patras, Rion, GR-26504, Greece

${ }^{5}$ Department of Imaging Physics, The University of Texas MD Anderson Cancer Center, Houston, TX, USA

${ }^{6}$ Institute of Mechanics and Advanced Materials, Cardiff School of Engineering, Cardiff University, Wales, UK

\section{Introduction}

Rapid assessment of blood flow in cerebral vascular diseases during neurovascular interventions is important for medical treatment planning. Unfortunately, estimation of local hemodynamic conditions in a given patient relies on the physicians' subjective judgment of dynamic contrast agent distribution patterns observed with Digital Subtraction Angiography (DSA).

Current DSA technology used in clinical practice cannot display volumetric blood flow rates. However, recent advancements, that include DSA with dual panel detector combined with deconvolution analysis, have started providing metrics of cerebral blood flow, cerebral blood volume and mean transit time. The major limitation of these noninvasive imaging techniques is the lack of temporal resolution.

Computational Fluid Dynamics (CFD) simulations provide quantitative assessment of the local hemodynamics. After the flow domain (vessel lumen) is segmented (usually with an efficient segmentation and edge detection algorithm), clinically relevant flow parameters can be computed. For the simulation results to be potentially useful in clinical practice, it is crucial to be able to obtain the flow field quickly and accurately. This can be achieved using the "image as a model" concept previously proposed in the context of creation of patient-specific 
computational biomechanics models for computing organ deformations for image guide surgery by Zhang et al. [1]. Using this concept, patient-specific computational grids are automatically generated directly from medical images.

In this study, the image as a model concept is implemented by representing the flow domain using a set or irregularly distributed nodes and uniform Cartesian embedded grid. The non-stationary Navier-Stokes equations in their velocityvorticity formulation are solved by using a meshless point collocation method. The spatial derivatives are computed with the recently developed meshless interpolation method referred to Discretization Corrected Particle Strength Exchange (DC PSE) [2]. For the transient term a $4^{\text {th }}$ order Runge-Kutta time integration scheme is used.

\section{Methods}

\subsection{Governing equations}

We consider the blood flow as non-stationary, viscous, laminar flow of an incompressible fluid. The governing equations for such flow are based on conservation of mass and momentum. The relevant non-dimensional form of the stream function-vorticity $(\psi-\omega)$ formulation [3] is:

$\frac{\partial \omega}{\partial t}+\frac{\partial \psi}{\partial y} \frac{\partial \omega}{\partial x}-\frac{\partial \psi}{\partial x} \frac{\partial \omega}{\partial y}=\frac{1}{R e} \nabla^{2} \omega$

$\nabla^{2} \psi=-\omega$

where $R e$ is the Reynolds number. The stream function $\psi$ can be used to compute the velocity components $u$ and $v$, using the following equations:

$u=\frac{\partial \psi}{\partial y}$

$v=-\frac{\partial \psi}{\partial x}$

We numerically solve Eqs. (1) and (2), defined in the spatial domain $\Omega$ with boundary $\partial \Omega$, having initial conditions

$\boldsymbol{u}=\boldsymbol{u}_{0}$

$\omega=\omega_{0}=\boldsymbol{\nabla} \times \boldsymbol{u}_{0}, \quad t=0$ 
and boundary conditions

$\boldsymbol{u}=\boldsymbol{u}_{\partial \Omega}$

$\omega=\left(\boldsymbol{\nabla} \times \boldsymbol{u}_{0}\right)_{\partial \Omega}$

\subsection{Meshless Discretization: Discretization Corrected Particle Strength Exchange Method}

We solve the partial differential equations (PDEs) governing the blood flow using a Discretization Corrected Particle Strength Exchange (DC PSE) method. The DC PSE was introduced in [4] as a Lagrangian based particle solution method. The authors in [2] have reformulated DC PSE method to work in the Eulerian framework required for applications in fluid flow. For completeness of the presentation, we provide a brief description of the Particle Strength Exchange (PSE) operators and the DC PSE interpolation method below.

\subsubsection{Particle Strength Exchange (PSE) operators}

The Particle Strength Exchange (PSE) method makes use of kernels to create approximate differential operators that guarantee the conservation of particle strength in particle-particle interactions. It was first introduced in 1989 by Degond and Mas-Gallic [5] for diffusion and convection-diffusion problems. Based on this initial work, Eldredge et al. [6] developed a framework for approximating arbitrary derivatives. In general, a PSE operator $Q^{\boldsymbol{\beta}} f(\boldsymbol{x})$ for approximating the derivative $D^{\boldsymbol{\beta}} f(\boldsymbol{x})$ has the form

$Q^{\boldsymbol{\beta}} f(\boldsymbol{x})=\frac{1}{\varepsilon^{|\boldsymbol{\beta}|}} \int(f(\boldsymbol{y}) \mp f(\boldsymbol{x})) \eta_{\varepsilon}^{\boldsymbol{\beta}}(\boldsymbol{x}-\boldsymbol{y}) d \boldsymbol{y}$

with $\eta_{\varepsilon}^{\boldsymbol{\beta}}=\eta^{\boldsymbol{\beta}}(x / \varepsilon) / \varepsilon^{d}$ being a scaled kernel of radius $\varepsilon$ and, $d$ being the number of dimensions. For conservation reasons, the sign in Eq. (7) is negative when $|\boldsymbol{\beta}|$ is even and positive when $|\boldsymbol{\beta}|$ is odd, with $\boldsymbol{\beta}$ a multi-index [5]. The challenge is to find a kernel $\eta_{\varepsilon}^{\boldsymbol{\beta}}$ that leads to accurate approximations for $D^{\boldsymbol{\beta}}$. To find such kernels for arbitrary derivatives, the idea in [6] is adopted, starting from the Taylor expansion of a function $f(\boldsymbol{y})$ about a point $\boldsymbol{x}$ :

$f(\boldsymbol{y})=f(\boldsymbol{x})+\sum_{|\boldsymbol{a}|=1}^{\infty} \frac{1}{\boldsymbol{a} !}(\boldsymbol{y}-\boldsymbol{x})^{\boldsymbol{a}} D^{\boldsymbol{a}} f(\boldsymbol{x})$

By introducing the continuous $\boldsymbol{\alpha}$-moments 
$M_{a}=\int(x-y)^{\alpha} \eta^{\beta}(x-y) d y=\int z^{\alpha} \eta^{\beta}(z) d z$

we obtain:

$Q^{\boldsymbol{\beta}} f(\boldsymbol{x})=\frac{(-1)^{|\boldsymbol{\beta}|}}{\boldsymbol{\beta} !} M_{\boldsymbol{\beta}} D^{\boldsymbol{\beta}} f(\boldsymbol{x})+\sum_{\substack{|\boldsymbol{a}|=1 \\ \boldsymbol{a} \neq \boldsymbol{\beta}}}^{\infty} \frac{(-1)^{|\boldsymbol{a}|}}{\boldsymbol{a} !} \varepsilon^{|\boldsymbol{a}|-|\boldsymbol{\beta}|} M_{\boldsymbol{a}} D^{\boldsymbol{a}} f(\boldsymbol{x})$

Finally, to approximate $Q^{\beta} f(\boldsymbol{x})$ with order of accuracy $r$, the following set of conditions is imposed for the moments $M_{a}$ :

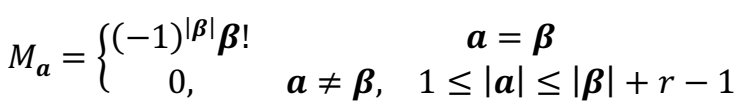

In addition, if we impose

$\int|\mathbf{z}|^{|\boldsymbol{\beta}|+r}\left|\eta^{\boldsymbol{\beta}}(\mathbf{z})\right| d \mathbf{z}<\infty$

the mollification error $\epsilon_{\varepsilon}(\boldsymbol{x})=D^{\boldsymbol{\beta}} f(\boldsymbol{x})-Q^{\boldsymbol{\beta}} f(\boldsymbol{x})$ is bounded [6]. The challenge is to construct a kernel that satisfies the conditions described in Eq. (10). A possible procedure has been described in [6]. Once the kernel is defined, the operator in Eq. (8) can be discretised using a midpoint quadrature over the nodes as

$Q_{h}^{\boldsymbol{\beta}} f(\boldsymbol{x})=\frac{1}{\varepsilon^{|\boldsymbol{\beta}|}} \sum_{p \in N(\boldsymbol{x})}\left(f\left(\boldsymbol{x}_{p}\right) \mp f(\boldsymbol{x})\right) \eta_{\varepsilon}^{\boldsymbol{\beta}}\left(\boldsymbol{x}-\boldsymbol{x}_{p}\right) V_{p}$

where $N(\boldsymbol{x})$ is the number of all nodes in a neighborhood around $\boldsymbol{x}$, which is usually defined by a cutoff radius $\boldsymbol{r}_{e}$, chosen such that $N(\boldsymbol{x})$ coincides to a certain level of accuracy with the kernel support. $V_{p}$ is the volume associated with each particle. Given such a discretization, the discretization error $\epsilon_{h}(\boldsymbol{x})=Q^{\boldsymbol{\beta}} f(\boldsymbol{x})-Q_{h}^{\boldsymbol{\beta}} f(\boldsymbol{x})$ is also bounded [6].

\subsubsection{The Discretization Corrected PSE operators}

The Discretization Corrected PSE (DC PSE) operators were introduced in [4], to reduce the discretization error $\epsilon_{h}(x)$ in the PSE operator approximation. The derivation of the DC PSE approximation starts from Eq. (13) (with $V_{p}$ included in the kernel function), with the requirement of finding a kernel function which minimizes the difference between this specific discrete operator and the actual derivative. To achieve this, each term $f\left(\boldsymbol{x}_{p}\right)$ in Eq. (13) is replaced with its Taylor 
expansion about $\boldsymbol{x}$, leading to the following expression for the derivative approximation:

$Q_{h}^{\boldsymbol{\beta}} f(\boldsymbol{x})=\frac{(-1)^{|\boldsymbol{\beta}|}}{\boldsymbol{\beta} !} Z_{h}^{\boldsymbol{\beta}} D^{\boldsymbol{\beta}} f(\boldsymbol{x})+\sum_{\substack{|\boldsymbol{a}|=1 \\ \boldsymbol{a} \neq \boldsymbol{\beta}}}^{\infty} \frac{(-1)^{|\boldsymbol{a}|}}{\boldsymbol{a} !} \varepsilon^{|\boldsymbol{a}|-|\boldsymbol{\beta}|} Z_{h}^{\boldsymbol{a}} D^{\boldsymbol{a}} f(\boldsymbol{x})+r_{0}$

with

$r_{0}=\left\{\begin{array}{cl}0, & |\boldsymbol{\beta}| \text { even } \\ 2 e^{-|\boldsymbol{\beta}|} Z_{h}^{0} f(\boldsymbol{x}) & |\boldsymbol{\beta}| \text { odd }\end{array}\right.$

and the discrete moments defined as

$Z_{h}^{a}=\frac{1}{\varepsilon^{d}} \sum_{p \in N(x)}\left(\frac{x-x_{p}}{\varepsilon}\right)^{a} \eta^{\beta}\left(\frac{x-x_{p}}{\varepsilon}\right)$

Therefore, the set of moment conditions become

$Z_{h}^{a}=\left\{\begin{array}{cc}(-1)^{|\boldsymbol{\beta}|} \boldsymbol{\beta} ! & \begin{array}{c}\boldsymbol{a}=\boldsymbol{\beta} \\ 0\end{array} \\ <\infty & \boldsymbol{a} \neq \boldsymbol{\beta} \\ <\infty \text { otherwise }\end{array} \quad a_{\text {min }} \leq|\boldsymbol{a}| \leq|\boldsymbol{\beta}|+r-1\right.$

for all $|\boldsymbol{\beta}| \neq 0$, where $a_{\min }$ is one when $|\boldsymbol{\beta}|$ is odd and 0 when $|\boldsymbol{\beta}|$ is even. The kernel $\eta^{\boldsymbol{\beta}}$ is chosen as:

$\eta^{\boldsymbol{\beta}}(\boldsymbol{x}, \mathbf{z})=\left(\sum_{|\boldsymbol{\gamma}|=a_{\min }}^{|\boldsymbol{\beta}|+r-1} a_{\boldsymbol{\gamma}}(\boldsymbol{x}) \mathbf{z}^{\gamma}\right) e^{-|\boldsymbol{z}|^{2}}=P(\boldsymbol{x}, \boldsymbol{z}) W(\mathbf{z}), \quad \boldsymbol{z}=\frac{\boldsymbol{x}-\boldsymbol{x}_{p}}{\varepsilon}$

The kernel function consists of a polynomial correction function $P(\boldsymbol{x}, \boldsymbol{z})$ and the weight function $W(\mathbf{z})$. Different choices for the weight function applied can be found in [6]. The unknown coefficients $a_{\gamma}(\boldsymbol{x})$ are obtained by requesting the kernel given by Eq. (18) to satisfy the conditions in Eq. (8). Given our choice of kernel function, the DC PSE derivative approximation becomes:

$$
\begin{aligned}
Q_{h}^{\beta} f\left(\boldsymbol{x}_{p}\right)=\frac{1}{\varepsilon\left(\boldsymbol{x}_{p}\right)^{\boldsymbol{\beta}}} \sum_{x_{q} \in \mathcal{N}\left(x_{p}\right)}\left(f\left(\boldsymbol{x}_{q}\right)\right. \\
\left.\mp f\left(\boldsymbol{x}_{p}\right)\right) \boldsymbol{p}\left(\frac{\boldsymbol{x}-\boldsymbol{x}_{p}}{\varepsilon\left(\boldsymbol{x}_{p}\right)}\right) \boldsymbol{a}^{T}\left(\boldsymbol{x}_{p}\right) e^{-\left(\frac{\left|x_{p}-\boldsymbol{x}_{q}\right|}{\varepsilon\left(\boldsymbol{x}_{p}\right)}\right)^{2}},
\end{aligned}
$$

where $\boldsymbol{p}(\boldsymbol{x})=\left[p_{1}(\boldsymbol{x}), p_{2}(\boldsymbol{x}), \ldots, p_{m}(\boldsymbol{x})\right]$, with $m$ being the number of monomial used ( $m=6$ and 10 for second order monomials in two and three dimensions, respectively) 
and, $\boldsymbol{a}(\boldsymbol{x})$ are the vectors of terms in the monomial basis and their coefficients, respectively.

\section{Runge-Kutta (RK4) explicit solver}

Given the vorticity $\omega^{(n)}$ and stream function values $\psi^{(n)}$ on the nodes, the updated values $\left\{\omega^{(n+1)}, \psi^{(n+1)}\right\}$ can be computed by:

$$
\begin{aligned}
& \frac{\omega^{(1)}-\omega^{(n)}}{\frac{1}{2} \Delta t}+\frac{\partial \psi^{(n)}}{\partial y} \frac{\partial \omega^{(n)}}{\partial x}-\frac{\partial \psi^{(n)}}{\partial x} \frac{\partial \omega^{(n)}}{\partial y}=\frac{1}{R e} \nabla^{2} \omega^{(n)}, \quad \nabla^{2} \psi^{(1)}=\omega^{(1)} \\
& \frac{\omega^{(2)}-\omega^{(n)}}{\frac{1}{2} \Delta t}+\frac{\partial \psi^{(1)}}{\partial y} \frac{\partial \omega^{(1)}}{\partial x}-\frac{\partial \psi^{(1)}}{\partial x} \frac{\partial \omega^{(1)}}{\partial y}=\frac{1}{R e} \nabla^{2} \omega^{(1)}, \quad \nabla^{2} \psi^{(2)}=\omega^{(2)} \\
& \frac{\omega^{(3)}-\omega^{(n)}}{\Delta t}+\frac{\partial \psi^{(2)}}{\partial y} \frac{\partial \omega^{(2)}}{\partial x}-\frac{\partial \psi^{(2)}}{\partial x} \frac{\partial \omega^{(2)}}{\partial y}=\frac{1}{R e} \nabla^{2} \omega^{(2)}, \quad \nabla^{2} \psi^{(3)}=\omega^{(3)} \\
& k_{4}=-\Delta t\left(\frac{\partial \psi^{(3)}}{\partial y} \frac{\partial \omega^{(3)}}{\partial x}-\frac{\partial \psi^{(3)}}{\partial x} \frac{\partial \omega^{(3)}}{\partial y}-\frac{1}{R e} \nabla^{2} \omega^{(3)}\right) \\
& \omega^{(n+1)}=\frac{1}{3}\left(-\omega^{(n)}+\omega^{(1)}+2 \omega^{(2)}+\omega^{(3)}\right)+\frac{1}{6} k_{4}, \quad \nabla^{2} \psi^{(n+1)}=\omega^{(n+1)}
\end{aligned}
$$

In each stage, updated values for the velocity are computed through Eq. (3a) and Eq. (3b). Meshless methods are recognized as one of the most accurate and efficient numerical methods to compute derivatives on an irregularly distributed set (cloud) of nodes. For the stream function-vorticity N-S equations, given the velocity field values computed previously by the updated stream function values $\psi^{(n+1)}$, we can compute the updated vorticity values $\omega^{(n+1)}$ for the entire spatial domain (including boundaries) by using the strong form meshless operators for first order spatial derivative as

$\omega^{(n+1)}=\frac{\partial v^{(n+1)}}{\partial x}-\frac{\partial u^{(n+1)}}{\partial y}$.

\section{Results}

We obtain the flow domain from brain Digital Subtraction Angiography (DSA) images (University Hospital Rion, Patras, Greece) (Fig. 1), with $128 \times 128$ pixel resolution. For the test case examined, the flow domain downstream forms a dilation (aneurysm) and bifurcates. 


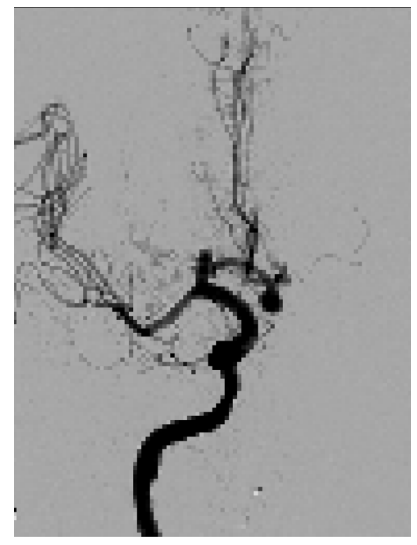

(a)

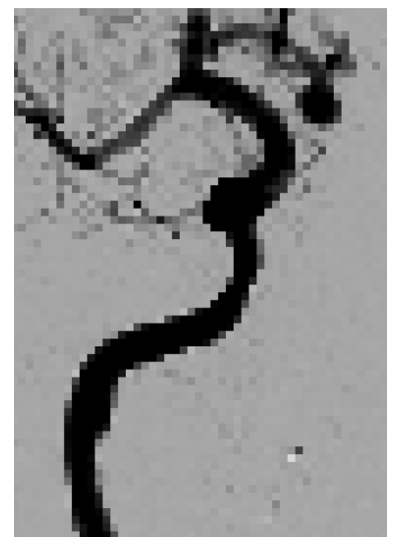

(b)

Figure 1. (a) DSA image and (b) region of interest (ROI).

The boundaries of the flow domain are obtained by thresholding segmentation and an edge detection method. In the context of meshless methods, the spatial domain is represented (not discretized like in mesh-based methods) by an irregular point cloud or a Cartesian embedded grid (Fig. 2). In both cases, generating the point cloud is a straightforward procedure. In the former, irregular nodes can be generated by using a standard 2D triangular mesh generator (we use only the nodes and not their connectivity). 2D mesh generators are fully automated and they are routinely used to discretize complex geometries. Additionally, there is no need to generate 'good quality mesh' an consequently, 'good quality point clouds'. In the latter, uniform Cartesian grid nodes are generated and use for the simulation only those located inside the flow domain.

For the test cases examined in the present study, we use in total 396,945 nodes (ensure a grid independent numerical solution) to represent the flow domain. We use the 2D triangular mesh generator (MESH2D-Delaunay-based unstructured mesh-generation). The time needed to create the irregular nodal distribution was not more that 5 seconds (computations were conducted in a Mac Pro laptop with 16 GB RAM and Intel i7 quad core $2.7 \mathrm{GHz}$ processor). For the uniform Cartesian grid, we use the build-in MATLAB functions to create the Cartesian grid and define the nodes located in the flow domain. Again the computation took no more than 5 seconds. 


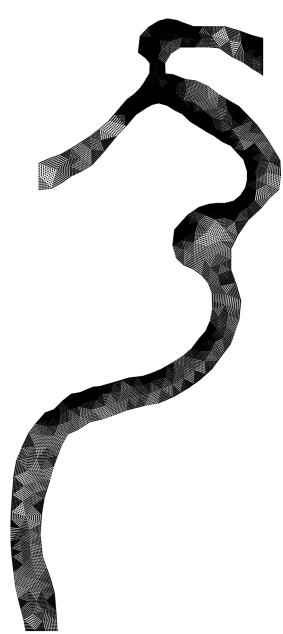

(a)

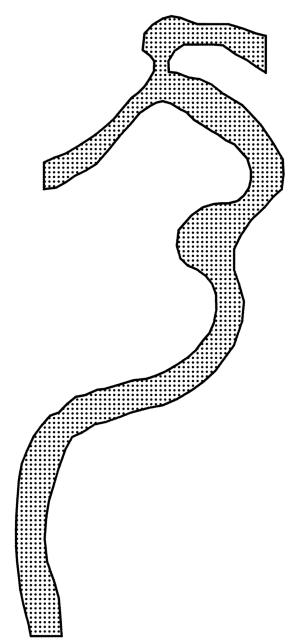

(b)

Figure 2. Representation of the flow domain with (a) irregular nodal distribution and (b) Cartesian embedded grid.

For the meshless point collocation method, spatial derivatives (up to $2^{\text {nd }}$ order) were computed with an in-house $\mathrm{C}++$ code (derivatives were computed in 3.2 seconds). The dynamic viscosity is set to $\mu=0.00032 \mathrm{~kg} / \mathrm{m} \mathrm{s}$ and the density to $\varrho=1050 \mathrm{~kg} / \mathrm{m}^{3}$. Flow simulation strongly depends on the boundary conditions, inflow and outflow boundary conditions. We have examine the robustness of the proposed scheme on the inflow boundary variability (different Reynolds number). We highlight, that the method performs efficient and is not altered by the variability of the inflow boundary conditions.

\section{Dependence on inflow boundary conditions}

Flow characteristics play an important role in vascular disease since they are heavily dependent on hemodynamic factors. As pointed-out earlier, measurement of in vivo hemodynamics can be difficult and in the majority of the cases invasive [7]. Computational fluid dynamics (CFD) is an approach able to quantify hemodynamics with high spatial and temporal resolution [8]. Due to the complex characteristics of the cardiovascular system, quantification of the intricate flow, velocity and pressure fields in vascular models can only be achieved by performing patient-specific simulations.

Accurate boundary conditions for CFD studies rely on a number of assumptions, such as the blood rheology, the computational grid size and adaptivity. Typically, in the majority of the studies idealized velocity profiles (such as a parabolic, plug 
or Womersley) for the inflow boundary conditions have been used, by measuring only a few in vivo velocity parameters such as peak velocity, average velocity, or flow rate [9]. The need for accurate inflow boundary conditions is particularly important for realistic and clinically useful flow results. Therefore, it might be expected that use of idealized velocity profiles, which do not take into account patient specific flow conditions and geometry alterations, would be inappropriate for patient specific hemodynamics study, and yield inaccurate CFD results.

In the present study, we demonstrate the efficiency of the proposed scheme to work with different inlet boundary conditions. We therefore consider parabolic and plug velocity profiles and examined the efficiency of the scheme and the alterations in the velocity magnitude may result in different flow regimes downstream. For the plug velocity profile, we consider two velocity magnitudes, namely $U_{m}=0.0015$ and $0.025 \mathrm{~m} / \mathrm{s}$, while in the case of parabolic and parabolic transient we apply $U_{\text {inlet }}=4 U_{m} x\left(H-x / H^{2}\right)$ and $U_{\text {inlet }}=4 U_{m} x\left(H-x / H^{2}\right) \sin (\pi t / 3)$, respectively, with $H$ being the inlet length. Reynolds number $R e=\rho U_{m} H / \mu$, with $D$ being a characteristic length. At both outlets, the flow is fully developed $(d u / d x=0)$ and, for the rest of the boundaries we applied no-slip conditions. The total time for the simulation was set to $T_{\text {tot }}=3 \mathrm{sec}$ and the time step used was $d t=10^{-4} \mathrm{sec}$ (the critical time step computed was $d t_{\text {critical }}=10^{-3} \mathrm{sec}$ ). Fig. 2 shows the nodal distributions, irregular and Cartesian embedded.

Fig. 3 shows the iso-contours for the stream function at $t=1 \mathrm{sec}$. We observe that when velocity inlet velocity increases, vortices are formed downstream, in the region of the aneurysm and close to the bifurcation.

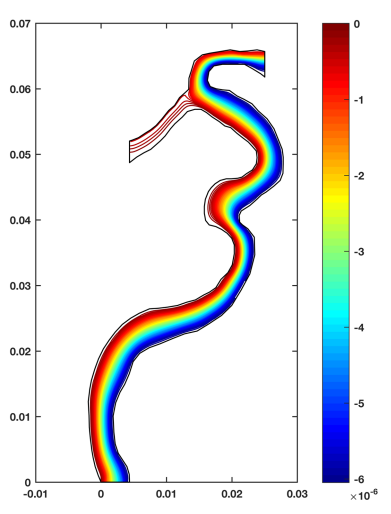

(a)

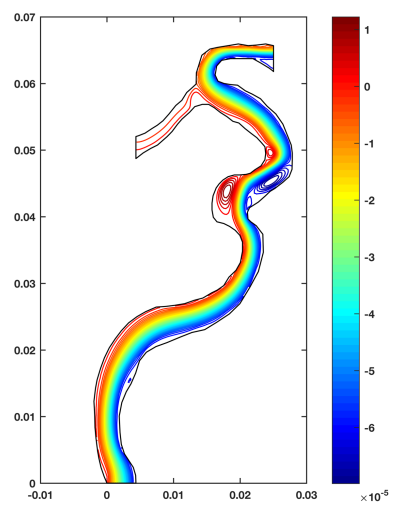

(b) 


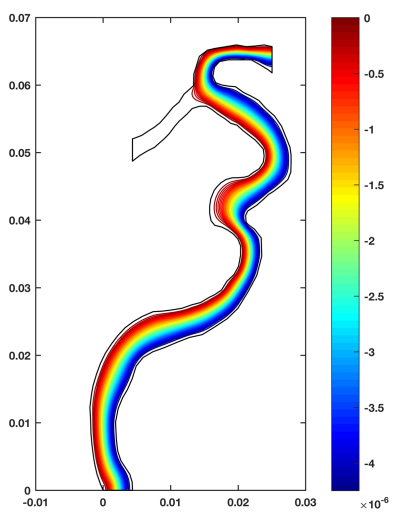

(c)

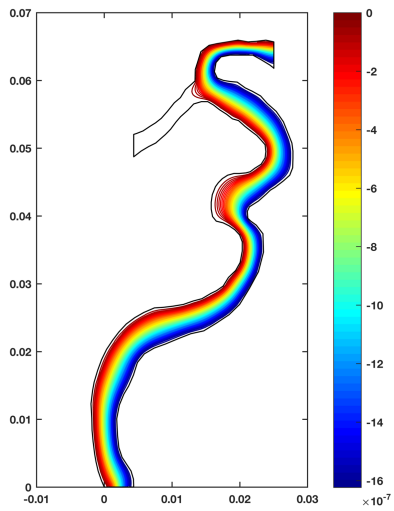

(e)

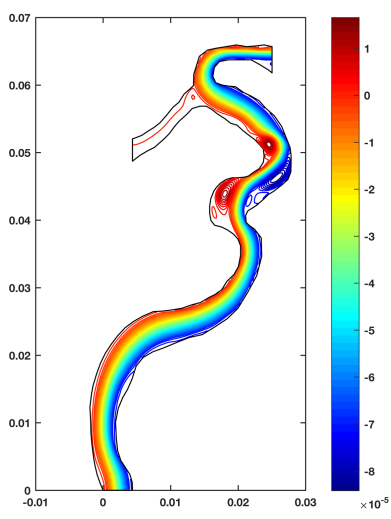

(d)

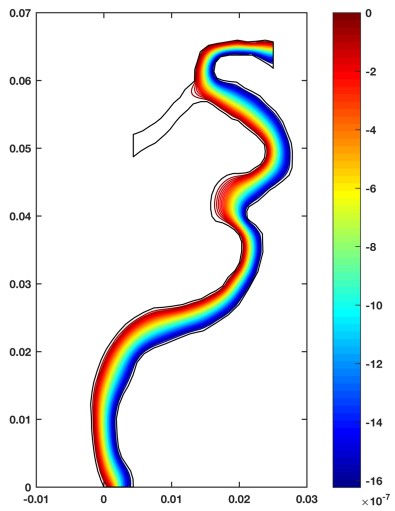

(f)

Figure 3. Predicted streamlines for $U_{m}=0.0015 \mathrm{~m} / \mathrm{s}$ (left) and $U_{m}=0.025 \mathrm{~m} / \mathrm{s}$ (right) (a)-(b) plug profile (c)-(d) parabolic profile and (e)-(f) parabolic unsteady profile (spatial dimensions are in meters).

\section{Discussion}

We have described a meshless point collocation algorithm for solving the nonstationary, incompressible, laminar Navier-Stokes equations over complex geometries of the vascular system.

We have tested the proposed algorithm using a flow domain derived from Digital Subtraction Angiography (DSA) images and we have examined the dependency of the flow field on the boundary conditions. The numerical results indicate that the method is efficient (computational time of around $5 \mathrm{~s}$ using a laptop for a model with close to 400,000 nodes) and facilitates straightforward treatment of different 
boundary conditions. The method appears to be efficient for both uniform Cartesian (embedded) grids and for irregular point clouds (see Fig. 3).

\section{Acknowledgements}

This research was supported by the Australian Government through the Australian Research Council's Discovery Projects funding scheme (project DP160100714).

\section{References}

1. Zhang, Joldes Wittek, Miller 2013 in International Journal for Numerical Methods in Biomedical Engineering

2. G. C. Bourantas, B. L. Cheesman, R. Ramaswamy, I. F. Sbalzarini. Using DC PSE operator discretization in Eulerian meshless collocation methods improves their robustness in complex geometries. Computers \& Fluids 136 285-300 (2016).

3. C. A. J. Fletcher. Computational Techniques for Fluid Dynamics, Vol I and II. Springer Series in Computational Physics. Springer, 1988.

4. Schrader, B., Reboux, S., Sbalzarini, I. F., Discretization correction of general integral PSE operators for particle methods. Journal of Computational Physics 229, 4159-4182 (2010).

5. Degond, P., Mas-Gallic, S. The weighted particle method for convectiondiffusion equations. Part 2: The anisotropic case. Mathematics of Computation 53 (188), 509-525 (1989).

6. Eldredge, J. D., Leonard, A., Colonius, T. A general deterministic treatment of derivatives in particle methods. Journal of Computational Physics 180 (2), 686-709 (2002).

7. Xiong, G. , Figueroa, C. A. , Xiao, N. , and Taylor, C. A. , 2011, " Simulation of Blood Flow in Deformable Vessels Using Subject-Specific Geometry and Spatially Varying Wall Properties,” Int. J. Numer. Method Biomed. Eng., 27(7), pp. 1000-1016.

8. Milner, J. S. , Moore, J. A. , Rutt, B. K. , and Steinman, D. A. , 1998, “ Hemodynamics of Human Carotid Artery Bifurcations: Computational Studies With Models Reconstructed From Magnetic Resonance Imaging of Normal Subjects,” J. Vasc. Surg., 28(1), pp. 143-156

9. Campbell, I. C. , Ries, J. , Dhawan, S. S. , Quyyumi, A. A. , Taylor, W. R. , and Oshinski, J. N. , 2012, “Effect of Inlet Velocity Profiles on Patient-Specific Computational Fluid Dynamics Simulations of the Carotid Bifurcation,” ASME J. Biomech. Eng., 134(5), p. 051001 